\title{
Two- and three-dimensional computation of solitary wave runup on non-plane beach
}

\author{
B. H. Choi ${ }^{1}$, E. Pelinovsky ${ }^{2}$, D. C. Kim ${ }^{1}$, I. Didenkulova ${ }^{2,3}$, and S.-B. Woo ${ }^{4}$ \\ ${ }^{1}$ Dept. of Civil \& Environmental Engineering, Sungkyunkwan Univ., Chunchun-dong 300, Jangan-gu, Suwon 440-746, Korea \\ ${ }^{2}$ Dept. of Nonlinear Geophysical Processes, Inst. of Applied Physics, 46 Uljanov Street, Nizhny Novgorod 603950, Russia \\ ${ }^{3}$ Inst. of Cybernetics, Tallinn Univ. of Technology, Akadeemia tee 21, 12618 Tallinn, Estonia \\ ${ }^{4}$ Dept. of Ocean Science, Inha Univ., 253 Yonghyun-dong, Nam-gu, Incheon 402-751, Korea
}

Received: 13 August 2007 - Revised: 3 January 2008 - Accepted: 28 May 2008 - Published: 24 June 2008

\begin{abstract}
Solitary wave runup on a non-plane beach is studied analytically and numerically. For the theoretical approach, nonlinear shallow-water theory is applied to obtain the analytical solution for the simplified bottom geometry, such as an inclined channel whose cross-slope shape is parabolic. It generalizes Carrier-Greenspan approach for long wave runup on the inclined plane beach that is currently used now. For the numerical study, the Reynolds Averaged Navier-Stokes (RANS) system is applied to study soliton runup on an inclined beach and the detailed characteristics of the wave processes (water displacement, velocity field, turbulent kinetic energy, energy dissipation) are analyzed. In this study, it is theoretically and numerically proved that the existence of a parabolic cross-slope channel on the plane beach causes runup intensification, which is often observed in post-tsunami field surveys.
\end{abstract}

\section{Introduction}

For the mitigation of tsunami hazard, the estimation of the characteristics of the flooding zone of the tsunami attack is one of the most important aspects of research. Analytical and numerical methods are widely applied to analyze the tsunami wave runup characteristics. Taking into account the usual large scales of tsunami waves induced by strong earthquakes, the depth-averaged 1-D and 2-D models of nonlinear shallow-water theory are used to study tsunami wave transformation and runup. Some of the numerical models (TUNAMI, MOST, etc.), adapted to the global ocean bathymetry datasets, are currently used in tsunami research (Goto et al., 1997; Titov and Gonzalez, 1997). Observed data, as well

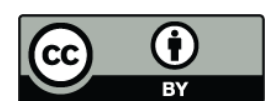

Correspondence to: E. Pelinovsky (enpeli@mail.ru) as data from laboratory experiments, are applied to verify the numerical models. The analytical approach, developed for the simplified beach geometry (plane beach of constant slope) and based on the Carrier-Greenspan transformation of shallow-water equations, is also applied to analyze tsunami runup characteristics (Yeh et al., 1997; Pelinovsky, 1997; Liu et al., 2007).

Real bottom bathymetry and coastal topography are much more complicated than the idealized plane beach. Runup of long waves on beaches of complicated bathymetry is studied numerically, mainly in the framework of shallow-water theory, see for instance (Zelt, 1986, Liu et al., 1995; OzkanHaller and Kirby, 1997; Titov and Synolakis, 1997; Brocchini et al., 2001). It is also the case that the wave field has large variability in temporal and spatial scales, especially with different near-shore conditions, where full 3-D flow characteristics appear. For these reasons, the existing analytical and numerical models are still not sufficient enough to explain many natural phenomena such as extreme runup height often observed in tsunami field surveys. For example, in 1993, southwest of Hokkaido, an earthquake in the Japan (East) Sea caused more than a 30-m runup height near Hamatsumae (Hokkaido Tsunami Survey Group, 1993). It is anticipated that this anomalously large runup height was mainly caused by the unique geometrical shape that is the existence of a channel on the beach whose cross-slope shape is parabolic in form. To explain such extreme runup phenomena, more accurate analytical and numerical models need to be developed, and some of the recent progress in this field is summarized in Dalrymple et al. (2006), Pelinovsky (2006) and Liu et al. (2007).

The main goal of this paper is to study the runup of solitary waves on non-plane beaches analytically and numerically. The rigorous analytical solution of the nonlinear shallowwater equations for the wave runup on the beach in a channel

Published by Copernicus Publications on behalf of the European Geosciences Union and the American Geophysical Union. 

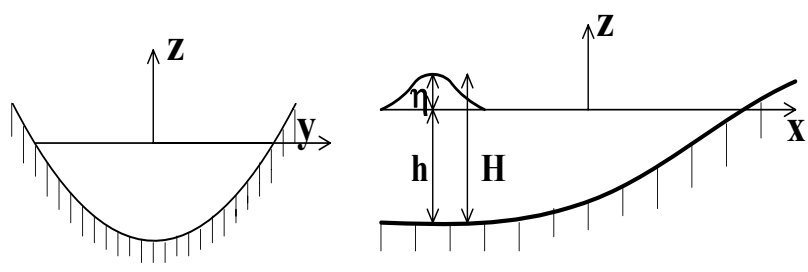

Fig. 1. The characterized cross-section and longitudinal projection of the bay.

of parabolic cross-slope shape is obtained. It generalizes the Carrier-Greenspan transformation that is currently used for the long wave runup on a plane beach of constant slope. It is theoretically shown that the runup height in the channel whose cross-slope shape is parabolic is larger than that of simple shaped cross-slope (Sect. 2). Numerical simulations of solitary wave runup on an inclined beach with parabolic cross-slope shape are performed in the framework of the 3D Reynolds Averaged Navier-Stokes system (Sect. 3). Detailed characteristics of the wave processes (water displacement, velocity field, turbulent kinetic energy and energy dissipation) are analyzed and compared. Results of 3-D computations are compared, particularly with the experimental data of Zelt (1986) reproduced in 2-D simulations by OzkanHaller and Kirby (1997), and analytical formulas for soliton runup.

\section{Analytical theory of long wave runup on beaches in a narrow bay of parabolic shape}

Usually tsunami waves generated by strong earthquakes have long wavelengths compared to water depth (in the order of hundreds of $\mathrm{km}$ ), and their fronts are almost straight (quasiplane waves). The characteristic width of the inclined channel we are interested in is significantly smaller than wave length (in the order of $10 \mathrm{~km}$ ), so that waves entering such channels are assumed to have uniform flow in the crosssection. For simplicity, we assume the analytical expression for the bottom shape as

$z(x, y)=-h(x)+f(y)$.

This geometry is displayed in Fig. 1. If the wave propagates along the $\mathrm{x}$-axis, the two-dimensional equations of nonlinear shallow water theory can be integrated on the cross-section, and the corresponding equations are one-dimensional:

$$
\frac{\partial u}{\partial t}+u \frac{\partial u}{\partial x}+g \frac{\partial H}{\partial x}=g \frac{d h}{d x}, \quad \frac{\partial S}{\partial t}+\frac{\partial}{\partial x}(S u)=0,
$$

where $H(x, t)=h(x)+\eta(x, t)$ is the total depth along the channel, $\eta(x, t)$ is the displacement of the water surface, $S(x, t)$ is the area of the cross-section of the channel, and $u(x, t)$ is the mean flow velocity. Integration of (1) makes the system (2) closed, and the solution depends on the beach geometry. The general approach to solve analytically the system (2) is suggested in (Zahibo et al., 2006). Here we consider a bay of parabolic shape

$f(y)=q y^{2}$,

therefore, the function, $S$ is

$S=\frac{4}{3 q^{1 / 2}} H^{3 / 2}$,

where $q$ is an arbitrary constant. The next approximation is a constant bottom slope of the channel axis; thus

$h(x)=-\alpha x$

The system (2) under these conditions reduces to

$$
\frac{\partial u}{\partial t}+u \frac{\partial u}{\partial x}+g \frac{\partial H}{\partial x}=g \frac{d h}{d x}, \quad \frac{\partial H}{\partial t}+u \frac{\partial H}{\partial x}+\frac{2}{3} H \frac{\partial u}{\partial x}=0,
$$

and differs from the "classical" one-dimensional equations for the wave runup on plane beach by the constant coefficient $2 / 3$. As a result, the same methods of solution of the hyperbolic system like the hodograph transformation can be applied for such geometry.

Introducing the Riemann invariants

$I_{ \pm}=u \pm 2 \sqrt{\frac{3}{2} g H}+\alpha g t$,

the system (6) is re-written in the form

$\frac{\partial I_{ \pm}}{\partial t}+c_{ \pm} \frac{\partial I_{ \pm}}{\partial x}=0$,

where the characteristic speeds are

$c_{ \pm}=\frac{2}{3} I_{ \pm}+\frac{1}{3} I_{\mp}-\alpha g t$.

Multiplying (8) on the Jacobian $\partial(t, x) / \partial\left(I_{+}, I_{-}\right)$, assuming that it is not zero (this value is achieved when the wave "breaks"), it can be transformed to

$\frac{\partial x}{\partial I_{\mp}}-c_{ \pm} \frac{\partial t}{\partial I_{\mp}}=0$.

The system (10) is nonlinear, due to the dependence $c_{ \pm}$from $I_{ \pm}$, but it can be reduced to linear by eliminating $x$

$\frac{\partial^{2} t}{\partial I_{+} \partial I_{-}}+\frac{2}{I_{+}-I_{-}}\left(\frac{\partial t}{\partial I_{-}}-\frac{\partial t}{\partial I_{+}}\right)=0$.

Let us introduce new arguments:

$\lambda=\frac{I_{+}+I_{-}}{2}=u+\alpha g t$,

$\sigma=\frac{I_{+}-I_{-}}{2}=\sqrt{6 g H}$. 
Then, Eq. (11) takes the form

$\frac{\partial^{2} t}{\partial \lambda^{2}}-\frac{\partial^{2} t}{\partial \sigma^{2}}-\frac{4}{\sigma} \frac{\partial t}{\partial \sigma}=0$

Extracting time from (12) and substituting

$u=\frac{1}{\sigma} \frac{\partial \Phi}{\partial \sigma}$

Eq. (14) is re-written in the final form

$\frac{\partial^{2} \Phi}{\partial \lambda^{2}}-\frac{\partial^{2} \Phi}{\partial \sigma^{2}}-\frac{2}{\sigma} \frac{\partial \Phi}{\partial \sigma}=0$.

In terms of the new variables, the physical variables can be expressed as

$\eta=\frac{1}{2 g}\left[\frac{2}{3} \frac{\partial \Phi}{\partial \lambda}-u^{2}\right], \quad u=\frac{1}{\sigma} \frac{\partial \Phi}{\partial \sigma}$,

$x=\frac{\eta}{\alpha}-\frac{\sigma^{2}}{6 g \alpha}, \quad t=\frac{\lambda-u}{g \alpha}$.

The Eq. (16) can be reduced to the 1-D wave equation, and its solution can be presented explicitly through two arbitrary functions

$\Phi(\lambda, \sigma)=\frac{\Psi_{1}(\lambda-\sigma)+\Psi_{2}(\lambda+\sigma)}{\sigma}$.

Taking into account that from physical point of view the function $\Phi$ should be bounded everywhere including the moving shoreline $\sigma=0$, and therefore only one arbitrary function $\Psi$ is in the solution (19). It transforms to

$\Phi(\lambda, \sigma)=\frac{\Psi(\lambda-\sigma)-\Psi(\lambda+\sigma)}{\sigma}$.

So, the initial set of nonlinear shallow water equations has the analytical solution (20) and all physical variables can be found via function $\Phi(\lambda . \sigma)$ using simple operations. The main advantage of this form is that the moving (unknown) shoreline now corresponds to $\sigma=0$ (since the total depth $H=0$ ) and therefore, solution (20) is determined in the halfline $-\infty<\sigma<0$ with fixed boundary. Such transformation generalizes the Carrier-Greenspan transformation (Carrier and Greenspan, 1958) actively used in the theory of long wave runup on plane beaches (Spielfogel, 1976; Pedersen and Gjevik, 1983; Synolakis, 1987; Pelinovsky and Mazova, 1992; Pelinovsky, 1996; Carrier et al., 2003; Kânoğlu, 2004; Tinti and Tonini, 2005; Kânoğlu and Synolakis, 2006; Didenkulova et al., 2006; Antuono and Brocchini, 2007). It is important to mention that in the case of the "parabolic" crossslope we have the simplified analytical solution to compare with the classical case of a plane slope beach when the general solution can be expressed in the integral form only. As a result, only algebraic manipulations are required to describe the tsunami wave runup on a beach. In fact, this approach for a parabolic bay was developed in the paper (Zahibo et al.,
2006) and is reproduced here for better understanding of the analytical solution obtained below.

The solution (20) has evident physical sense and describes the reflection of incident waves from the beach. In the case of non-breaking and non-dissipative waves the reflected wave has the same parameters as the incident wave, but opposite polarity. In this case where the incident wave is far from the beach where it is linear, it can be described by the linear expressions that follow from (17) and (18)

$\eta_{i n}(x, t)=\left.\frac{1}{3 g \sqrt{6 g h(x)}} \frac{\partial \Psi(\lambda-\sqrt{6 g h})}{\partial \lambda}\right|_{\lambda=g \alpha t}$,

where the argument of the incident wave is

$\lambda-\sqrt{6 g h(x)}=g \alpha\left[t-\sqrt{\frac{6|x|}{g \alpha}}\right]$,

and the last term presents the travel time in a basin of variable depth, $\int d x / c(x)$, where $c(x)=[2 g h(x) / 3]^{1 / 2}$ is the linear long wave speed in the parabolic channel. Wave amplitude is proportional to $h^{-1 / 2}$ according to the Green's law for a parabolic channel. Fixing the distance from the beach, $L$ or equivalent depth, $h_{0}=\alpha L$, we may determine the function $\Psi$ knowing the time series of the tsunami wave at this point. In particular, if the incident tsunami wave is a solitary wave

$\eta_{i n}(t)=A \operatorname{sech}^{2}\left[\frac{t-t_{0}}{T}\right]$

with arbitrary amplitude, duration and phase, the function $\Psi$ is

$\Psi(\lambda)=3 g^{2} \alpha T A \sqrt{6 g h_{0}} \tanh \left[\frac{\lambda-\lambda_{0}}{g \alpha T}\right]$

and the solution (20) becomes fully determined. The calculation of the wave field (water elevation and particle velocity) requires algebraic manipulations using (17) and (18).

Here we consider the runup characteristics only. The dynamics of the moving shoreline are given by (17) and (18) at $\sigma=0$, thus the vertical displacement and velocity of the shoreline can be expressed as a function of time by parametric curves

$$
\begin{aligned}
& R(\lambda)=\eta(\lambda, 0)=\frac{1}{2 g}\left[\frac{2}{3} \frac{\partial \Phi}{\partial \lambda}-u^{2}\right], \\
& u(\lambda, 0)=\frac{1}{\sigma} \frac{\partial \Phi}{\partial \sigma}, \quad t=\frac{\lambda-u(\lambda, 0)}{g \alpha},
\end{aligned}
$$

where $\Phi$ is determined by (20) and $\Psi$ by (24). In particular, if the amplitude of the incident wave is small, we may neglect nonlinear terms in (25). Also, from (20), on the shoreline $(\sigma \rightarrow 0)$, it follows that $\Phi(\lambda, \sigma=0)=-2 \partial \Psi / \partial \lambda \sim \eta_{\text {inc }}$, (see Eq. 21). As a result, the simplified formula for the vertical 


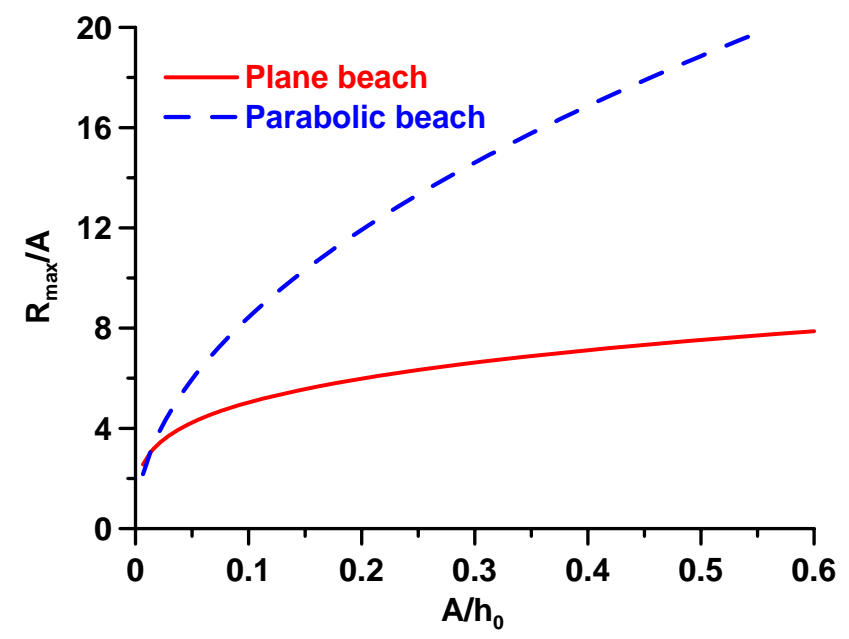

Fig. 2. Runup height of a solitary wave on a plane beach (solid red line) and a parabolic beach (dash blue line), slope $\alpha=0.1$.

displacement of the water level on a shore can be obtained from

$R(t)=-\frac{2 \sqrt{6} L}{\sqrt{g h_{0}}} \frac{d \eta_{i n}(t-\tau)}{d t}$,

where $\tau$ is the wave travel time from a fixed point to the shore. It is important to mention that oscillations at the shoreline end after the wave reflects. This differs from the case of a plane beach, where oscillations formally continue for infinite time. For moderate amplitudes the dynamics of the moving shoreline can be plotted from (25). However, it is easy to show that for any values of the incident wave amplitudes (in a non-breaking regime) the maximal values of runup and rundown can be found as extremes of the function (26) as in linear theory the velocities are too small in phases of high or low water (the same situation exists for a plane beach). As a result, the maximum runup height is calculated trivially for any shape of incident wave. In particular, when a solitary wave approaches to the beach, maximum vertical runup height is

$\frac{R_{\max }}{A}=\frac{16}{3 \sqrt{3}} \frac{L}{\lambda_{0}}$,

where $\lambda_{0}=c_{0} T$ is the characteristic soliton length, and $c_{0}=\left(2 g h_{0}\right) / 3^{1 / 2}$ is the linear speed of long waves in the parabolic channel. The formula (27) differs significantly from that in the case of the plane beach, where factor $L / \lambda$ has the exponent 1/2, (Pelinovsky and Mazova, 1992; Pelinovsky, 2006). In fact, formula (27) can be obtained for an incident wave of arbitrary shape and only the numeric coefficient will be changed. If we use as the initial wave the solitary wave solution of the Korteweg-de Vries equation for an even bottom

$\eta(x)=A \operatorname{sech}^{2}\left[\sqrt{\frac{3 A}{4 h_{0}}} \frac{x}{h_{0}}\right]$,

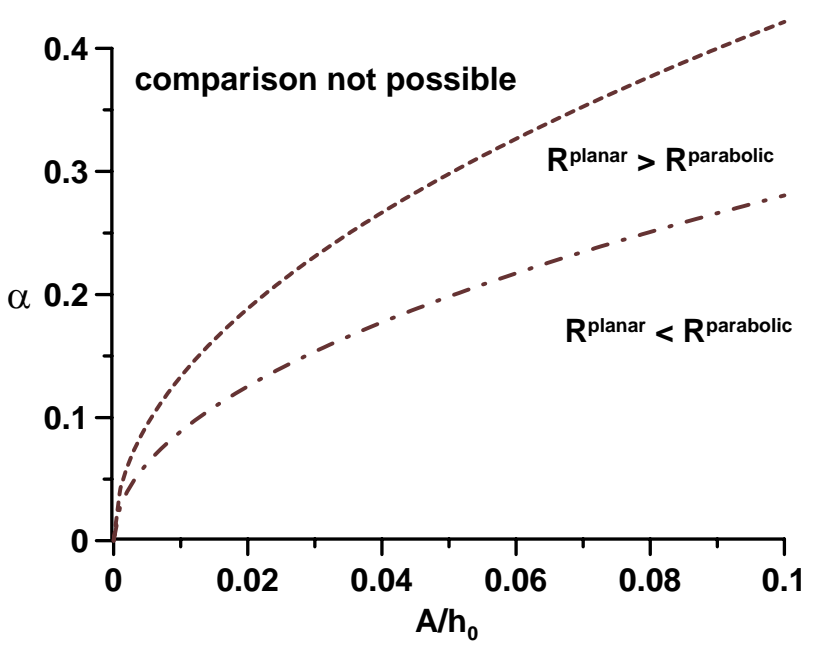

Fig. 3. Comparison of runup heights for planar and parabolic beaches.

the runup formula can be rewritten in the another form

$R_{\max }=\frac{8}{3} \frac{A}{\alpha} \sqrt{\frac{A}{h_{0}}} \sim A^{3 / 2}$

For comparison we also provide the similar formula for a plane beach (Synolakis, 1987)

$R_{\max }=2.8312 \frac{A}{\sqrt{\alpha}}\left(\frac{A}{h_{0}}\right)^{1 / 4} \sim A^{5 / 4}$.

Recently, Antuono and Brocchini (2007) found the highorder correction to (30). We should mention that formulas (29) and (30) are not valid for weak amplitudes of solitons, since the length of these solitons is large and comparable with the distance to the shore. In the limited case of a very large wavelength, the beach plays the role of a vertical wall, where $R_{\max } \approx 2 A$ (this result is evident if the plane beach is matched with a bottom of a constant depth). Pelinovsky (1996) and Madsen and Fuhrman (2008) demonstrated that the asymptotic expression (30) can be applied in the range of $R_{\max }>2 A$ for a plane beach. The same can be assumed for a parabolic beach. From the condition $R_{\max }=2 \mathrm{~A}$ follows the minimal value of the soliton amplitude when formulas (29) and (30) are valid

$\frac{A_{\min }}{h}=q \alpha^{2}$,

where $q=9 / 16=0.563$ for a parabolic beach, and $(2 / 2.8312)^{4}=0.249$ for a plane beach.

Figure 2 demonstrates the maximal runup height for two beach geometries: plane and parabolic beaches, with beach slope $\alpha=0.1$. The parabolic shape of the beach in this case leads to the concentration of wave energy near the beach and an increase in the runup height. A more detailed comparison of formulas (29) and (30), taking into account the limitation (31), is given in Fig. 3. It confirms that generally, the 
amplification on the parabolic beach is higher than on a plane beach, but for limited conditions of wave amplitude and bottom slope the runup height in a parabolic channel is less then on a plane beach.

Therefore, the rigorous solution of the nonlinear shallow water equations confirms the frequent observation that tsunami waves in bays with a decreasing cross-section are more energetic, and runup heights are usually larger than in basins with a constant cross-section.

\section{Three-dimensional runup computations}

The bottom geometry used in the numerical simulations is presented in Fig. 4. The numerical tank has the dimensions: length, $13 \mathrm{~m}$; width, $3.6 \mathrm{~m}$; height, $0.55 \mathrm{~m}$; water depth, $0.35 \mathrm{~m}$. At the end of the tank there is the inclined wall (slopes: $30^{\circ}, 45^{\circ}$ and $60^{\circ}$ ) containing a concaved semicylinder with radius $1.2 \mathrm{~m}$. This geometry should adequately demonstrate the "non-plane" effects during the runup process.

For detail computing of the solitary wave runup, the CFD code FLOW-3D developed by Hirt and Nichols (1981) is applied. The program solves the 3-D Reynolds-Averaged Navier-Stokes (RANS) equations with a free boundary. The details of the code are described in Flow-Science, 2002. In a previous study (Choi et al., 2007), we applied this code to study solitary wave runup on a conical island, and the model accuracy was verified by comparing the numerical results with the analytic solution and 2-D shallow water model results (Liu et al., 1995) for solitary wave propagation over constant depth. In this study three turbulent models $[k-\varepsilon$, RNG (Renormalization Group) theory, LES (Large Eddy Simulation)] are tested for solitary wave propagation and the models accuracies are compared. The model results are generally similar to each other, except that $k-\varepsilon$ results were slightly under estimated. LES model and RNG model results were largely similar, but the RNG computational cost was about $30 \%$ of the LES computation. This conclusion was consistent with the previous report, i.e., RNG model is known to describe more accurately low intensity turbulent flows. Therefore we choose the RNG model for the turbulent model, and other turbulent models are not considered in this study.

The computational domain (numerical tank) used in this study is described above. In total it contains 1069200 cells, 55 cells in the vertical direction, 72 cells in the longitudinal direction, and 270 cells in the transverse direction. The grid size in the vertical direction is $0.01 \mathrm{~m}$ and $0.20 \mathrm{~m}$ in the longitudinal direction. The grid size in the transverse direction is not constant with the minimum size being $0.015 \mathrm{~m}$.

The initial solitary wave height of $0.035 \mathrm{~m}$ (ratio amplitude/depth is 0.1 , so the wave nonlinearity is weak) enters at the incident wave boundary and it propagates along the channel. The procedure of the solitary wave generation by

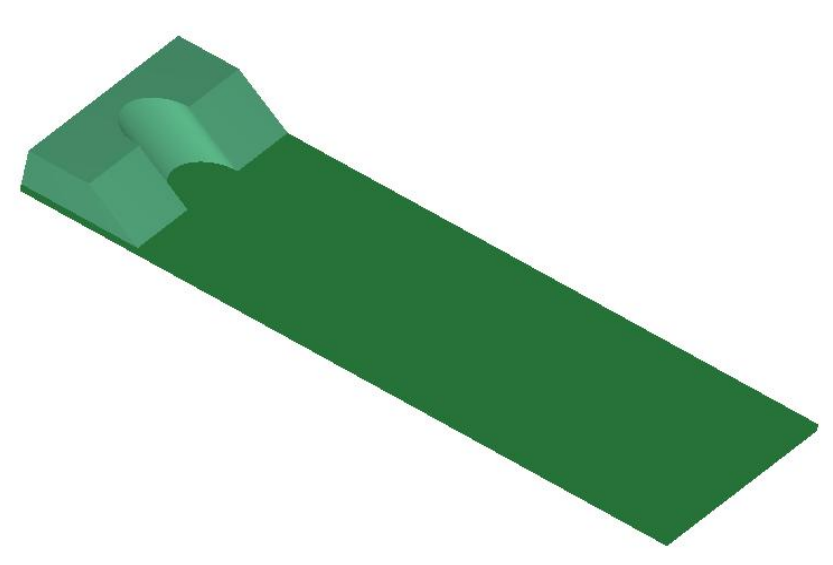

Fig. 4. Basin geometry for 3-D computing of solitary wave runup.

a piston wave maker is given by Katell and Eric (2002) and the application of wave maker theory to the FLOW-3D model simulation was tested and verified in the previous study (Choi et al., 2007). The shape of solitary wave is the solution of the Korteweg-de Vries Eq. (28).

Snapshots of the wave transformation and runup on the beach are illustrated in Fig. 5 for a beach slope of $30^{\circ}$. It is clearly seen that the wave height distribution along the front is almost cross-sectionally uniform when the wave is climbing up on the beach, and this is consistent with the 1$\mathrm{D}$ analytical theory developed in Sect. 2. It is seen that the wave runup at the central section (inner most part) is slightly smaller than the maximum wave height up to $t=7.5 \mathrm{~s}$ (Fig. 5a and b). At $t=8.7 \mathrm{~s}$, the runup height of $0.45 \mathrm{~m}$ at the innermost point in the channel is calculated (Fig. 5d) and it is $115 \%$ of the runup height at the beach $(0.39 \mathrm{~m})$. In Fig. $5 \mathrm{f}$, it is seen that the runup height at the inner-most point is slightly smaller than the reflected wave height at $t=9.5 \mathrm{~s}$, after it reaches its maximum height at $t=8.7 \mathrm{~s}$, In the reflected wave the diverged cylindrical field (diffracted field) on the "knife" edges is formed.

An enlargement of the "non-plane" structure of the runup front is shown in Fig. 6. Such effects can not be described in the framework of 1-D theory where the smoothness of the coastal line and bottom topography is assumed and in this case numerical study is more effective for the understanding of the physical process of wave runup at the parabolic crossslope shaped channel. Figure 6 clearly shows the geometrical effect on the wave runup intensification, i.e., the concaved channel plays a major role in wave energy focusing so that maximum wave height occurs at the inner-most point. As previously described, maximum runup height at the innermost point is $15 \%$ larger than that of slope when nonlinearity is 0.1 . This justifies the theoretical estimation.

Qualitatively, the general feature of wave runup for the wall slopes of $45^{\circ}$ and $60^{\circ}$ is almost the same as the case of wall slope of $30^{\circ}$, and corresponding figures are not displayed. Quantitatively, however, the bottom slope influences 


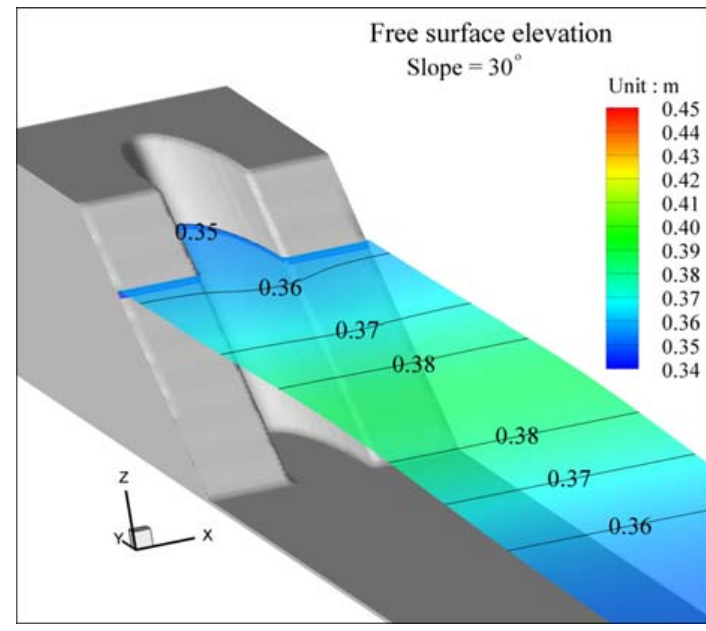

(a) $t=7.0 \mathrm{sec}$

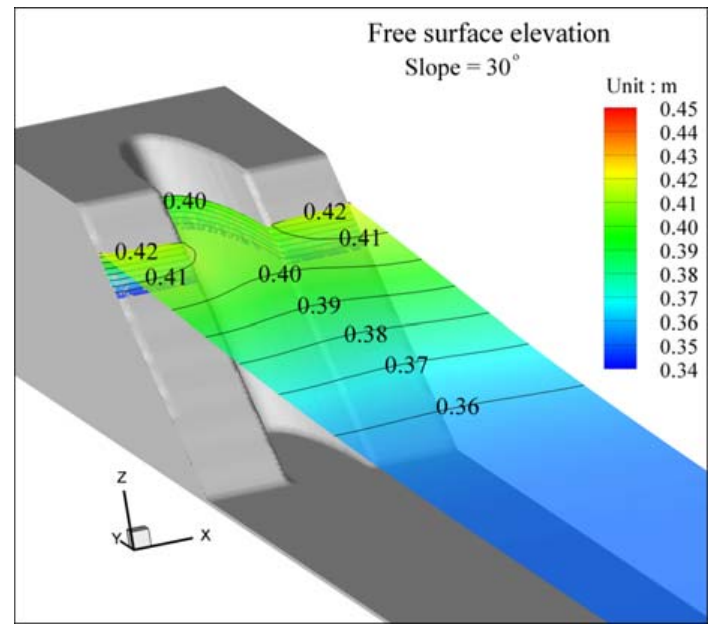

(c) $\mathrm{t}=8.0 \mathrm{sec}$

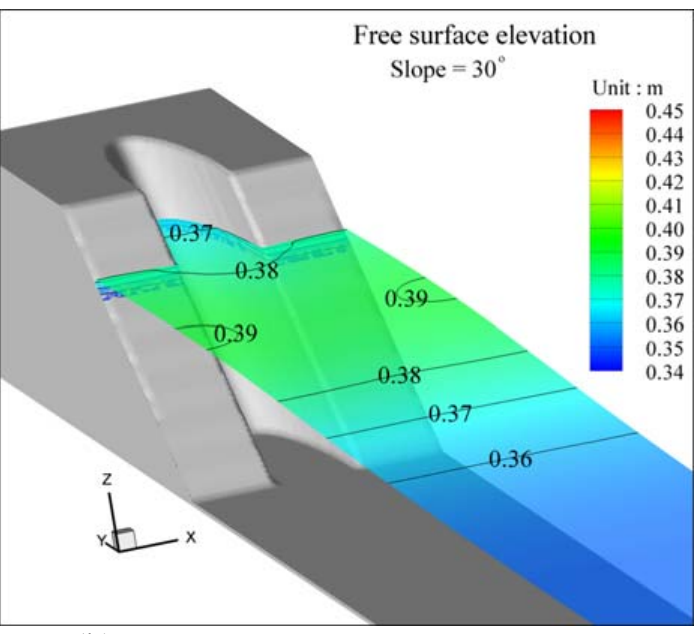

(b) $\mathrm{t}=7.5 \mathrm{sec}$

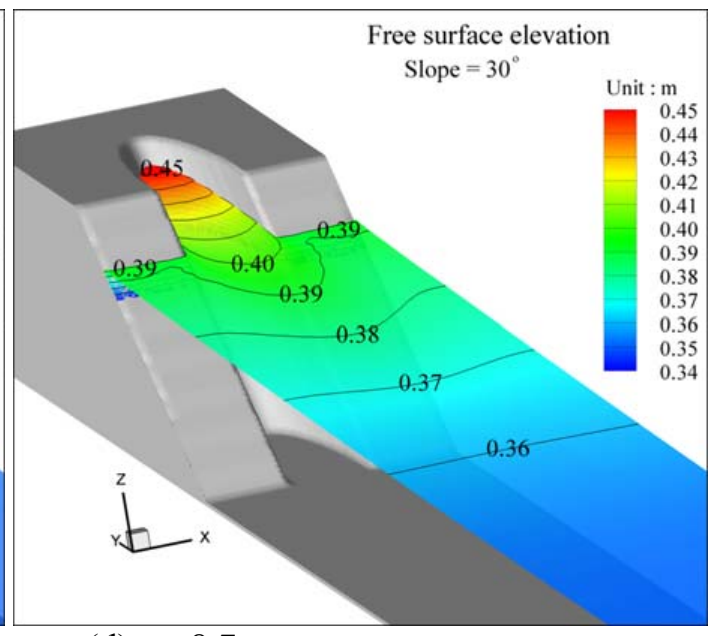

(d) $\mathrm{t}=8.7 \mathrm{sec}$

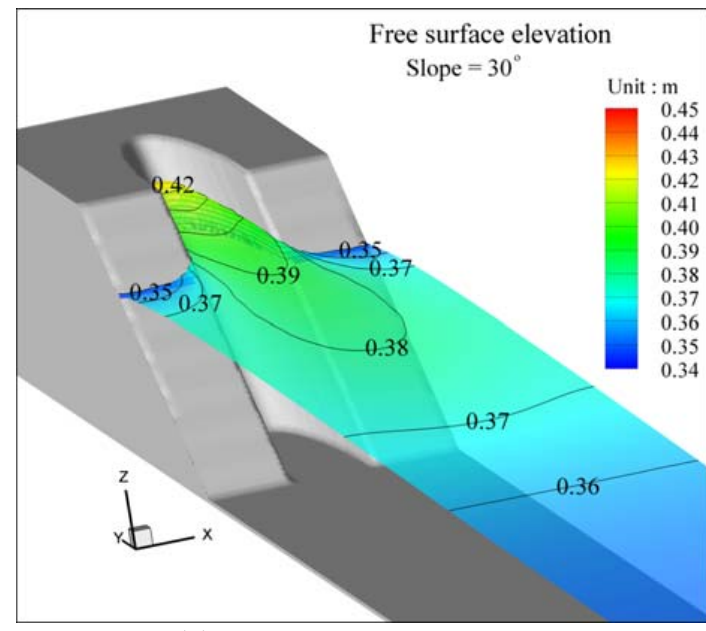

(e) $\mathrm{t}=9.0 \mathrm{sec}$

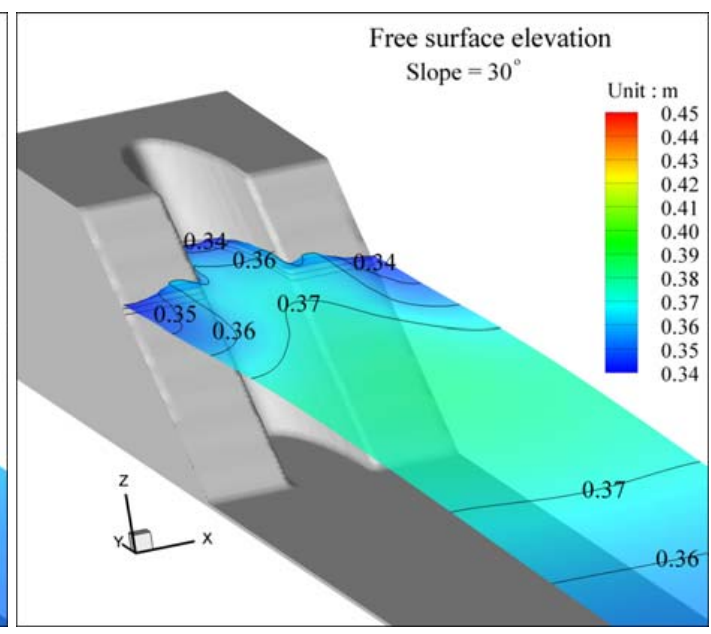

(f) $t=9.5 \mathrm{sec}$

Fig. 5. Contours of the sea level displacement near a concaved slope wall (slope: $30^{\circ}$ ). 


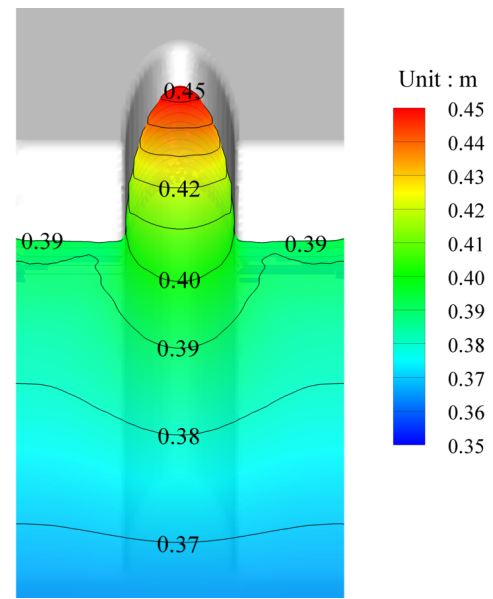

Fig. 6. Contours of the sea level displacement near concaved slope wall at $8.7 \mathrm{~s}$ (slope: $30^{\circ}$ ).

the wave height; see Fig. 7. As expected, the smaller the slope, the bigger the maximum particular, the maximum runup height is equal to $0.1055 \mathrm{~m}, 0.097 \mathrm{~m}$ and $0.0923 \mathrm{~m}$ for slopes runup height is. In $30^{\circ}, 45^{\circ}$ and $60^{\circ}$ respectively. However, according to the Synolakis's formula (30) for the plane beach the runup height is $0.077 \mathrm{~m}, 0.063 \mathrm{~m}$ and $0.054 \mathrm{~m}$ for the same slopes. The exceedance of the computed values above theoretical ones illustrates the importance of the "non-plane" geometry on the runup characteristics and the strong influence of local features of the coastal topography (up to $30 \%$ in height).

Figure 8 displays the spatial distribution of the velocity field from $7.5 \mathrm{~s}$, when the solitary wave approaches the front of island, to $9.5 \mathrm{~s}$, when the wave reflects from the beach. As it is clearly seen, the velocity distribution along the vertical coordinate is not uniform, and the velocity field is weaker in the bottom layer and higher near the sea surface. We mentioned this difference in (Choi et al., 2007), discussing the results of computing the wave runup on a conical island in the framework of the same model. The vertical velocity component is not weak at the runup stage (Fig. 8b-e), and it suggests the limitation of the previous model study based on shallow-water equations, where the vertical velocity components are assumed to be zero.

The wave flow is not uniform in the transverse direction either (Fig. 9). The runup in the concave channel is accompanied by the biggest flow velocities. The appearance of wave energy concentration in the channel during the water runup stage is very often observed in tsunami field surveys. In particular, this situation can explain the anomalous runup height of $30 \mathrm{~m}$ during the 1993 Okushiri tsunami (Hokkaido Tsunami Survey Group, 1993; Titov and Synolakis, 1997). The numerical solution demonstrates the non-monotonic dependence of the maximal velocity on the bottom slope and therefore the velocity of the wave runup on the almost ver-

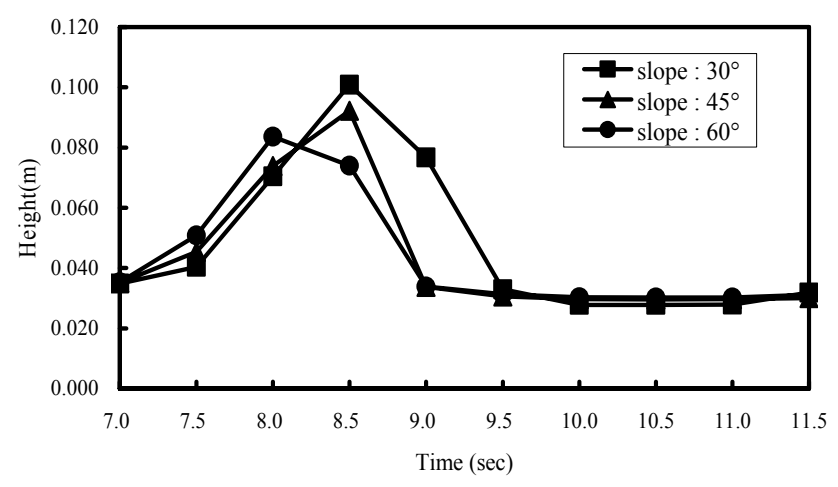

Fig. 7. Maximum wave height versus time.

tical wall can be higher. The same effect is manifested in a fully nonlinear non-hydrostatic 2-D case as pointed out in Cooker et al. (1997), Wood et al. (2000) and LonguetHiggins and Drazen (2002).

In Fig. 10, the role of the turbulent dissipation on the bottom boundary layer on the runup stage is demonstrated for the case of bottom slope of $45^{\circ}$, by presenting the turbulent kinetic energy (TKE in $\mathrm{m}^{2} / \mathrm{s}^{2}$ ) and the turbulent kinetic energy dissipation (DTKE in $\mathrm{m}^{2} / \mathrm{s}^{3}$ ) at the water surface The non-uniform spatial distribution pattern of turbulent energy is clearly seen. TKE is concentrated in the region where the water surface touches the solid bottom boundary.

In fact, the vertical distribution of the turbulent characteristics is not uniform either (Fig. 11) underlying the importance of the 3-D numerical models to describe the wave runup in basins with real topography. The temporal variation of the maximum values of these characteristics for a bottom slope of $45^{\circ}$ is presented in Fig. 12. Maximal turbulent motion appears at the moment of maximal runup of the solitary wave on the beach, when its energy increases by several orders. The same behavior is obtained for other bottom slopes. The maximum value of turbulent kinetic energy is $0.1 \mathrm{~m}^{2} / \mathrm{s}^{2}$ for slope $30^{\circ}, 0.03 \mathrm{~m}^{2} / \mathrm{s}^{2}$ for slope $45^{\circ}$, and $0.04 \mathrm{~m}^{2} / \mathrm{s}^{2}$ for slope $60^{\circ}$. Non-monotonic variations of turbulent kinetic energy, in principle, correlate with nonmonotonic behavior of the velocity in the climbing wave. The behavior of the turbulent kinetic energy dissipation is also non-monotonic $\left(1.39 \mathrm{~m}^{2} / \mathrm{s}^{3}\right.$ for slope $30^{\circ}, 1.46 \mathrm{~m}^{2} / \mathrm{s}^{3}$ for slope $45^{\circ}$, and $0.44 \mathrm{~m}^{2} / \mathrm{s}^{3}$ for slope $60^{\circ}$ ) but the maximum of DTKE is reached when TKE is at a minimum.

Zelt (1986) conducted a physical experiment for concavetype sloping bathymetry with maximum slope about $11.3^{\circ}$ and minimum slope $5.7^{\circ}$, with incoming solitary waves, and the experiment data are compared with model simulations results (Zelt, 1986; Ozkan-Haller and Kirby 1997). In this study, we apply the numerical model to the same physical experiment and show the three-dimensional snapshots as well as the time history of runup at several locations. Figure 13 shows the three-dimensional water surface snapshots when 


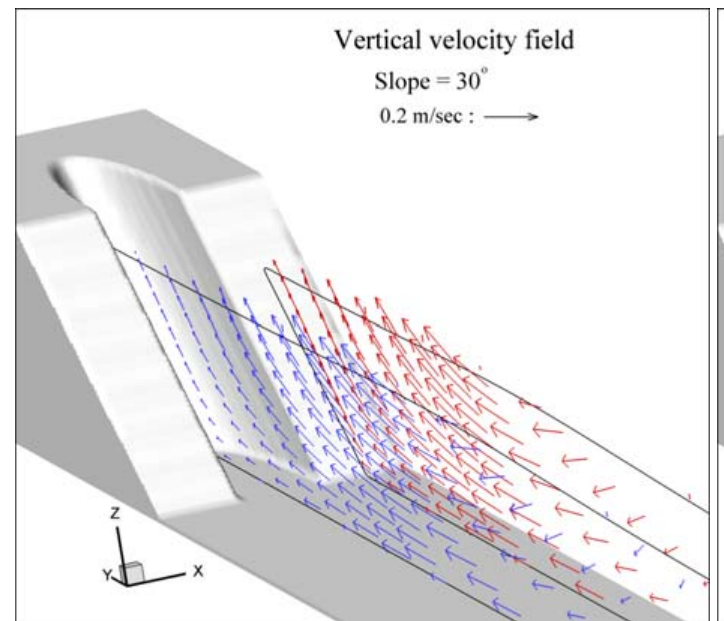

(a) $\mathrm{t}=7.0 \mathrm{sec}$

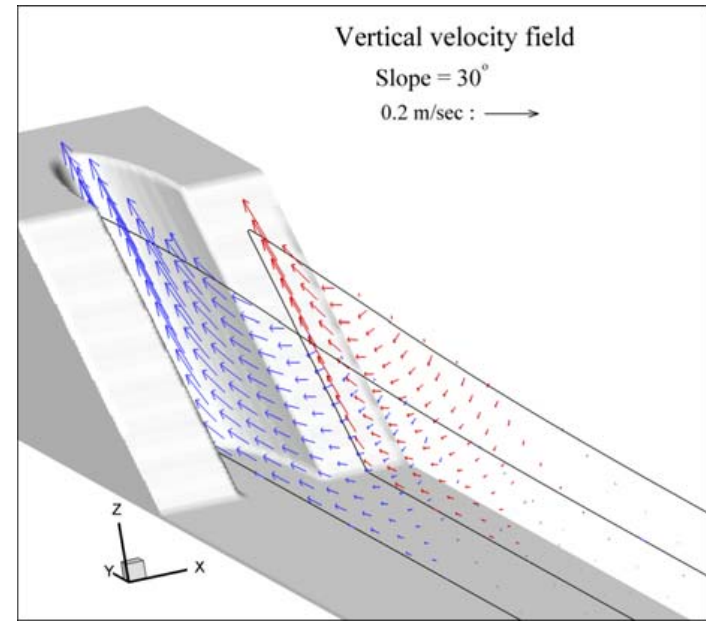

(c) $\mathrm{t}=8.0 \mathrm{sec}$

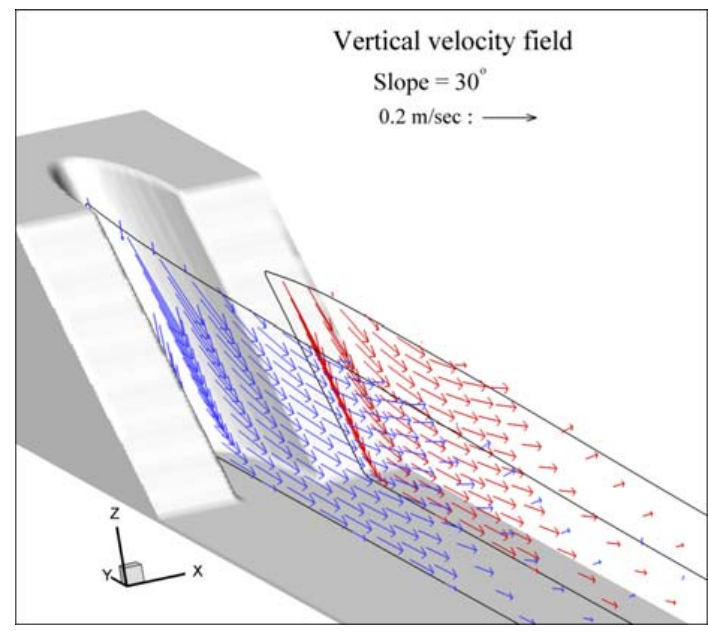

(e) $t=9.0 \mathrm{sec}$

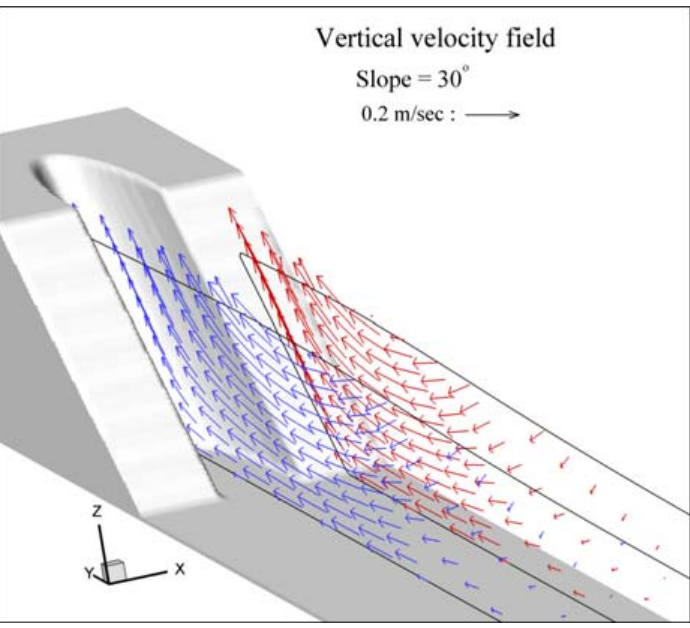

(b) $\mathrm{t}=7.5 \mathrm{sec}$

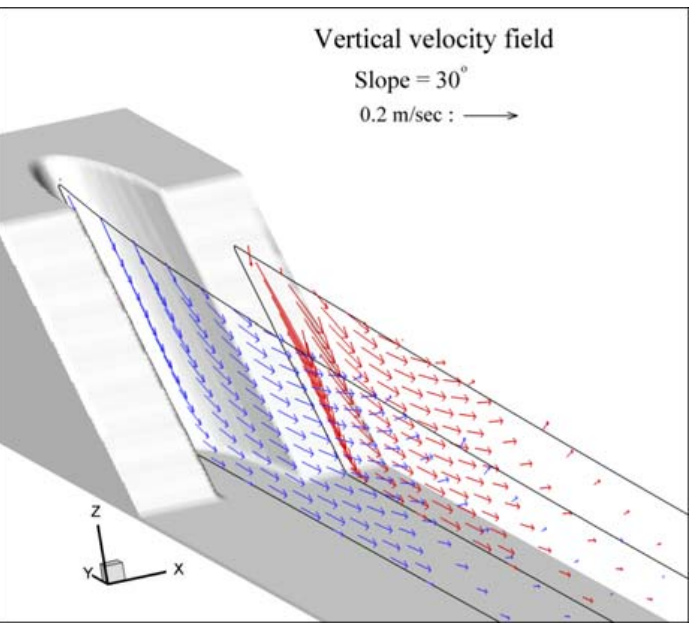

(d) $\mathrm{t}=8.7 \mathrm{sec}$

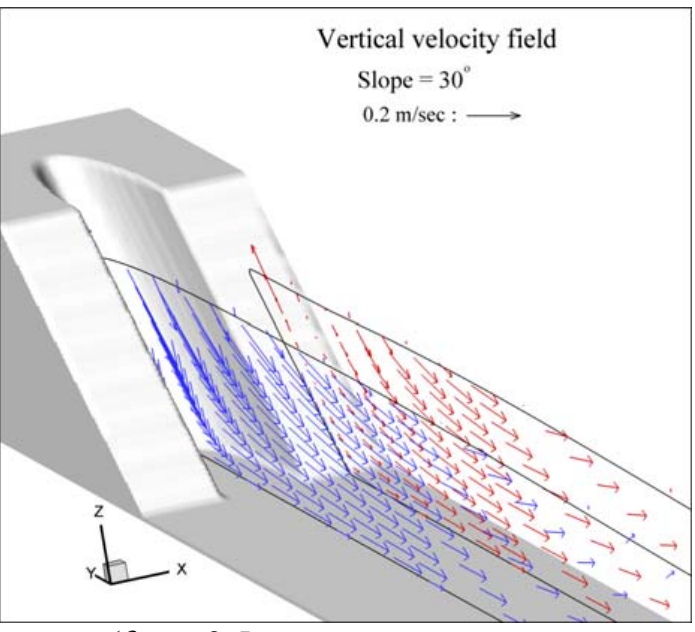

(f) $\mathrm{t}=9.5 \mathrm{sec}$

Fig. 8. Velocity distribution in the center of basin and at the periphery near the concave slope with slope: $30^{\circ}$. 
the runup reaches its maximum and minimum respectively. As shown in the figure, the shape of the runup at its maximum and minimum is quite different. At its maximum (Fig. 13a), the runup is focused at the center of the bathymetry and the surface slope is steep toward the center as illustrated by the contour of water surface. On the other hand, at its minimum (Fig. 13b) the runup is directed onshore and the surface slope is lower than at the maximum. It can be interpreted that the wave energy has focused at the center at the run-up stage and spread to the on/off shore direction at its run-down stage.

Figure 14 shows the normalized runup in the cross-shore direction as a function of non-dimensional time and space scales at different locations along the bay. In general, a good agreement is found between the physical experiment and numerical simulation in terms of water surface variation in time and space. These results verify the model accuracy and stability.

\section{Conclusions}

The runup of solitary waves on a "non-plane" beach is studied analytically and numerically. In the analytical study the nonlinear shallow water equations are solved rigorously for the parabolic cross-slope shaped bay using the generalized Carrier-Greenspan transformation. It is shown that the wave runup in basins with decreasing cross-sections leads to increase of the runup height, confirming many field observations of tsunami waves. More complicated coastal zone geometry (plane beach containing the concaved semi-cylinder) is studied numerically using the 3-D Reynolds averaged Navier-Stokes equations realized in FLOW-3D. The computed values of the runup heights for this geometry exceed the similar ones for a plane beach demonstrating the importance of local features of coastal topography in the process of wave runup. This fact has also been demonstrated for the one-dimensional case (Pelinovsky, 1996; Kanoglu and Synolakis, 1998), but for two-dimensional case it leads to big variations in wave amplitude. The flow velocity field is nonuniform in the vertical and transverse directions. The runup velocities in the concave area are bigger then on the periphery. The characteristics of the turbulent kinetic energy and the turbulent kinetic energy dissipation are computed also. They are also non-uniform in the vertical and transverse directions. The strong turbulent motion appears at the stage of the maximum wave runup. The values of the velocity, turbulent kinetic energy and turbulent kinetic energy dissipation are not monotonic functions of the bottom slope. Analytical and numerical results for beaches of different geometries show the importance of complicated seafloor bathymetry in the vicinity of the shoreline on runup characteristics, and quantitatively agree with tsunami observations in many areas of the world's ocean.

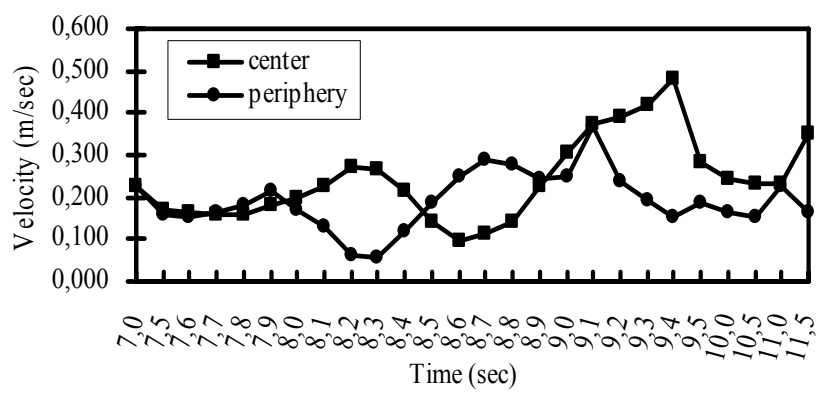

(a)

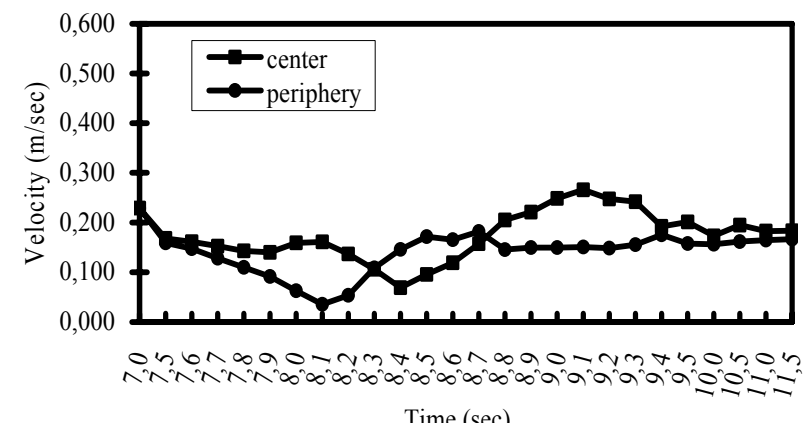

(b)

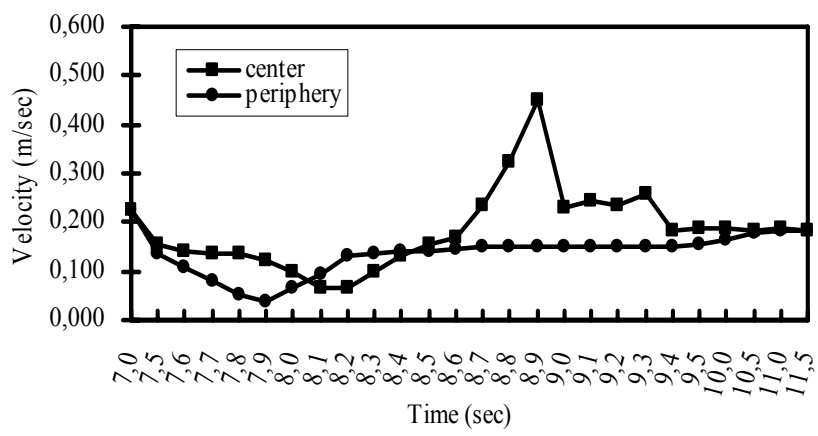

Fig. 9. Maximum velocity versus time for various slopes (a) slope: $30^{\circ}$ (b) slope: $45^{\circ}$ (c) slope: $60^{\circ}$. 

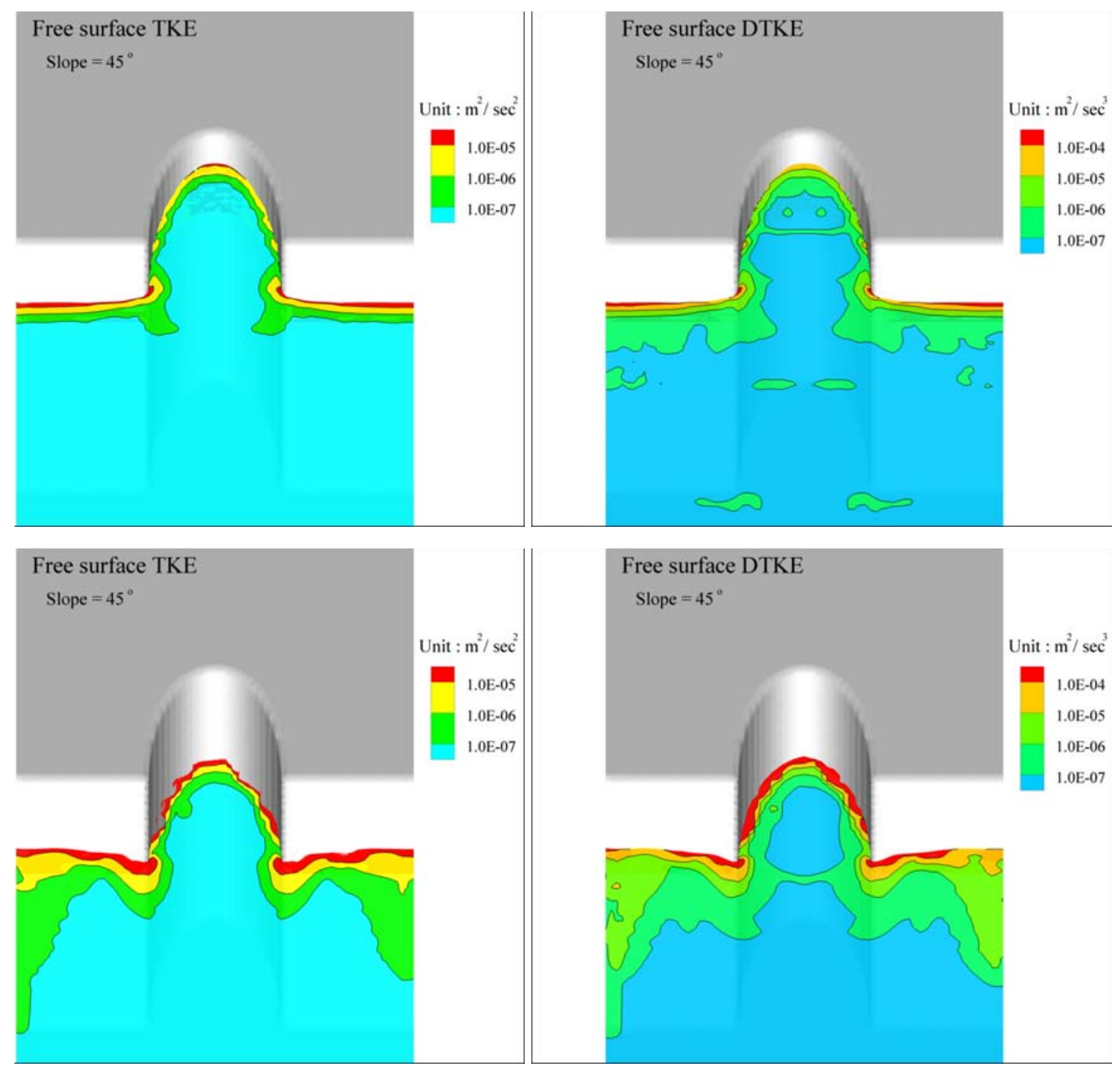

Fig. 10. Spatial distribution of turbulent kinetic energy (left) and turbulent kinetic energy dissipation (right) with slope $45^{\circ}$. 

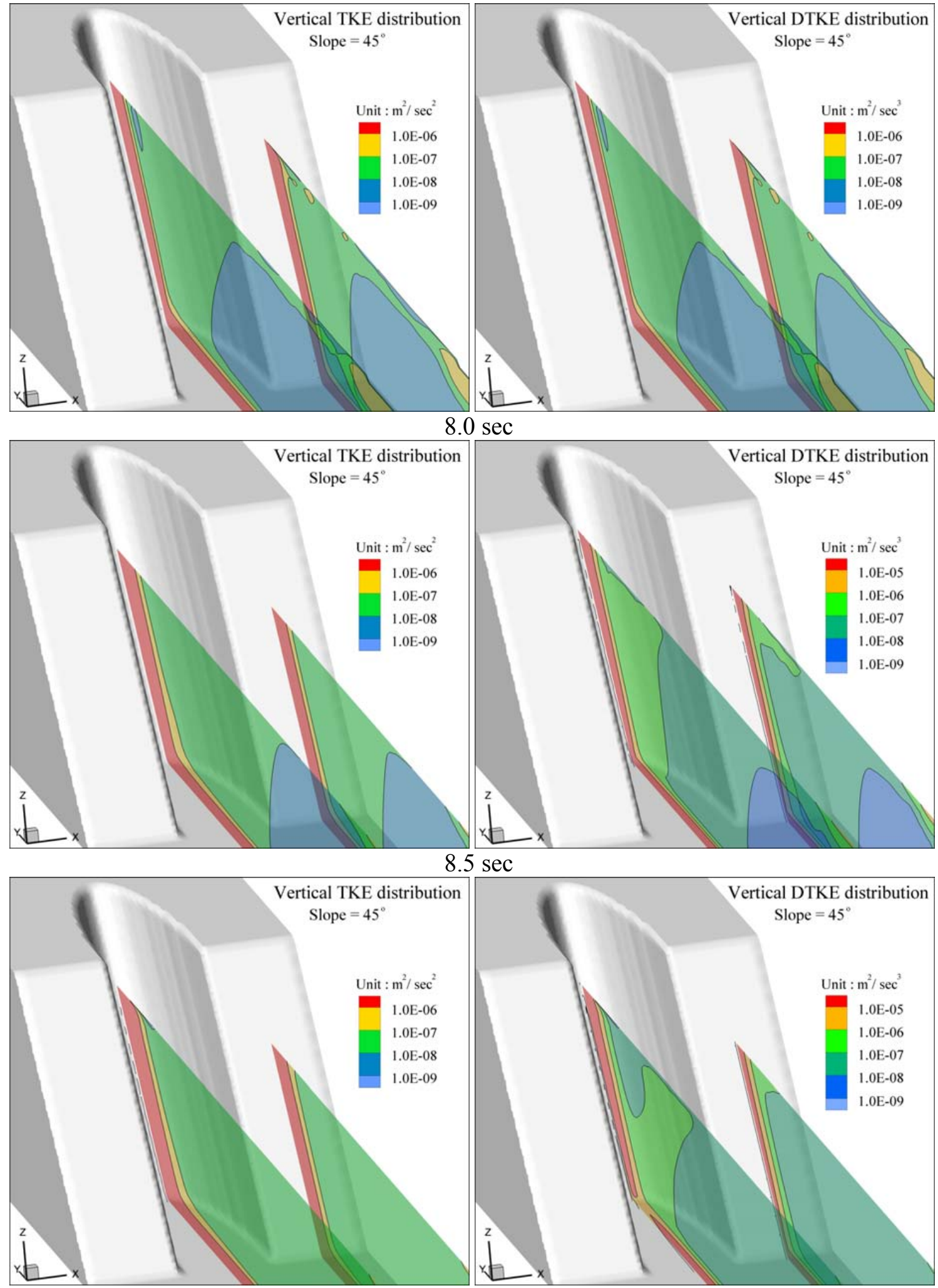

$9.5 \mathrm{sec}$

Fig. 11. Vertical distribution of turbulent kinetic energy (left) and turbulent kinetic energy dissipation (right) for slope $45^{\circ}$. 

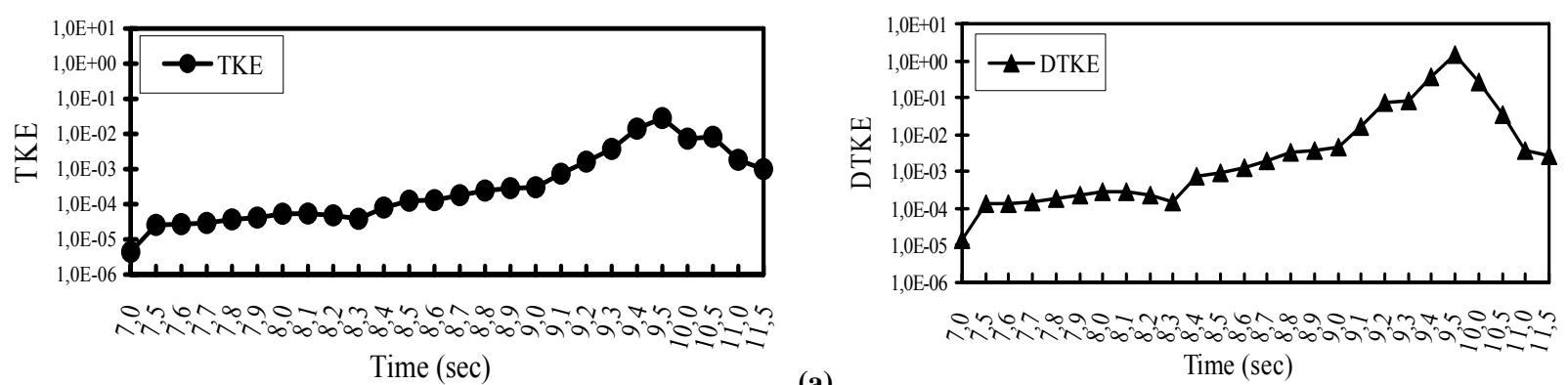

(a)

(b)

Fig. 12. Maximal values of turbulent kinetic energy (a) and turbulent kinetic energy dissipation (b) versus time for slope $45^{\circ}$.
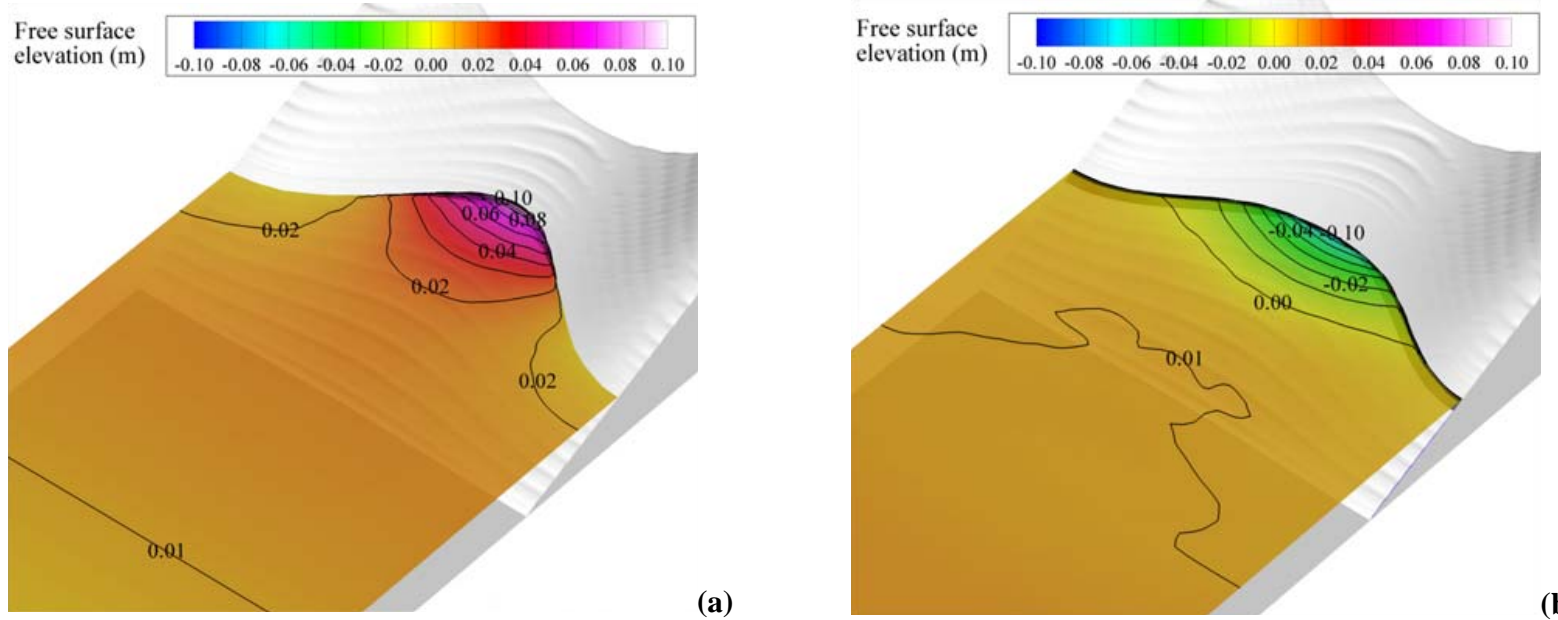

(a)

(b)

Fig. 13. Snapshots of the free surface distribution at its maximum (a) and minimum (b) at the central point.

Acknowledgements. Supports from Korean National Emergency Management Agency, INTAS (06-1000013-9236 and 06-10000146046), RFBR (06-05-72011 and 08-05-00069), Marie Curie network SEAMOCS (MRTN-CT-2005-019374) and Scientific School of V. Zverev are acknowledged. We would like to thank Prof. Kevin Parnell from James Cook University, Australia for useful comments.

Edited by: V. Shrira

Reviewed by: three anonymous referees

\section{References}

Antuono, M. and M. Brocchini, M.: The Boundary Value Problem for the Nonlinear Shallow Water Equations, Stud. Appl. Math., 119, 73-93, 2007.

Brocchini, M., Bernetti, R., Mancinelli, A., and Albertini, G.: An efficient solver for nearshore flows based on the WAF method, Coast. Eng., 43, 105-129, 2001.

Carrier, G. F. and Greenspan, H. P.: Water waves of finite amplitude on a sloping beach, J. Fluid Mech., 4, 97-109, 1958.

Carrier, G. F., Wu, T. T., and Yeh, H.: Tsunami run-up and drawdown on a plane beach, J. Fluid Mech., 475, 79-99, 2003.
Choi, B.-H., Kim, D. C., Pelinovsky, E., and Woo, S. B.: Threedimensional simulation of tsunami run-up around conical island, Coast. Eng., 54, 618-629, 2007.

Cooker, M. J., Weidman, P. D., and Bale, D. S.: Reflection of a high-amplitude solitary wave at a vertical wall, J. Fluid Mech., 342, 141-158, 1997.

Dalrymple, R. A., Grilli, S. T., and Kirby, J. T.: Tsunamis and challenges for accurate modelling, Oceanography, 19, 142-151, 2006.

Didenkulova, I. I., Zahibo, N., Kurkin, A. A., Levin, B. V., Pelinovsky, E. N., and Soomere, T.: Runup of nonlinearly deformed waves on a coast, Dokl. Earth Sci., 411, 1241-1243, 2006.

Flow Science Inc.: FLOW-3D User's Manual, 2002.

Goto, C., Ogawa, Y., Shuto, N., and Imamura, N.: Numerical method of tsunami simulation with the leap-frog scheme (IUGG/IOC Time Project), IOC Manual, UNESCO, 35, 1997.

Haller, H. T. and Kirby, J. T.: A Fourier-Chebyshev collocation method for the shallow water equations including shoreline runup, Appl. Ocean Res., 19, 21-34, 1997.

Hirt, C. W. and Nichols, B. D.: Volume of Fluid (VOF) method for the dynamics of free boundaries, J. Comp. Phys., 39, 201-225, 1981.

Hokkaido Tsunami Survey Group: Tsunami devastates Japanese coastal Regions, EOS, Trans. AGU, 74, 417-432, 1993.

Katell, G. and Eric, B.: Accuracy of solitary wave generation by a 


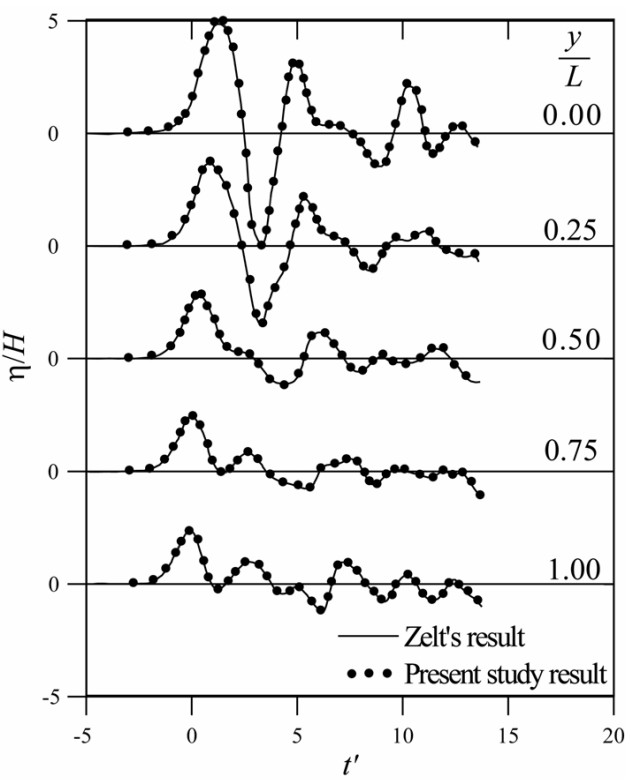

Fig. 14a. Temporal comparison of the model results and Zelt's experimental data for normalized runup height in the cross shore direction (incident wave height to offshore water depth ratio $H / h_{0}=0.02, h_{0}$ : offshore water depth, $H$ : incident wave height $=0.02 \mathrm{~m}, \eta$ : run-up height, $L$ : half-width of the bay, $t^{\prime}=t \sqrt{g h_{0}} / L$ ).
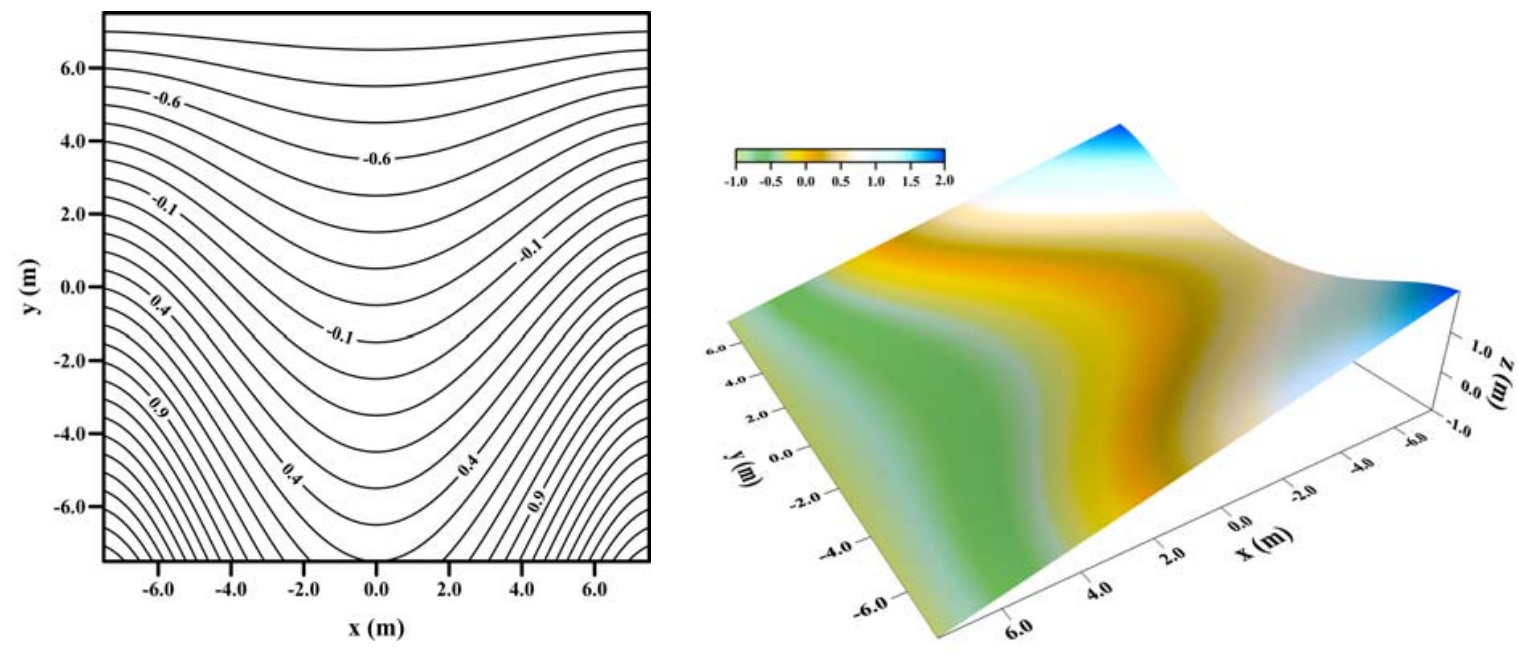

Fig. 14b. The bathymetry of the runup test with axis and orientation.

piston wave maker, J. Hydraul. Res., 4, 321-331, 2001.

Kânoğlu, U.: Nonlinear evolution and runup-rundown of long waves over a sloping beach, J. Fluid Mech., 513, 363-372, 2004.

Kânoğlu, U., and Synolakis, C.: Long wave runup on piecewise linear topographies, J. Fluid Mech., 374, 1-28, 1998.

Kânoğlu, U., and Synolakis, C.: Initial value problem solution of nonlinear shallow water-wave equations, Phys. Rev. Lett., 97, 148501, 2006.

Liu,P. L.-F., Cho, Y. S., Briggs, M. J., Kânoğlu, U., and Synolakis, C. E.: Run-up of solitary waves on a circular island, J. Fluid
Mech., 302, 259-285, 1995.

Liu, P. L.-F., Yeh, H., and Synolakis, C. (Eds.): Advanced numerical models for simulating tsunami waves and runup, Advances in Coastal and Ocean Engineering 10, World Scientific Publishing, Singapore, 2007.

Longuer-Higgins, M. S. and Drazen, D. A.: On steep gravity waves meeting a vertical wall: a triple instability, J. Fluid Mech., 466, 305-318, 2002.

Madsen, P. A. and Fuhrman, D. R.: Run-up of tsunamis and long waves in terms of surf-similarity, Coast. Eng., 55, 209-223, 
2008.

Pedersen, G. and Gjevik, B.: Run-up of solitary waves, J. Fluid Mech., 142, 283-299, 1983.

Pelinovsky, E. N.: Hydrodynamics of tsunami waves, Institute of Applied Physics Press, Nizhny Novgorod, 1996.

Pelinovsky, E.: Hydrodynamics of tsunami waves, in: "Waves in Geophysical Fluids", edited by: Grue, J. and Trulsen, K., CISM Courses and Lectures, 489, 1-48, Springer, 2006.

Pelinovsky, E. and Mazova, R.: Exact analytical solutions of nonlinear problems of tsunami wave run-up on slopes with different profiles, Nat. Hazards, 6, 227-249, 1992.

Spielfogel, L. O.: Run-up of single wave on a sloping beach, J. Fluid Mech., 74, 685-694, 1976.

Synolakis, C. E.: The runup of solitary waves, J. Fluid Mech., 185, 523-545, 1987.

Tinti, S. and Tonini, R.: Analytical evolution of tsunamis induced by near-shore earthquakes on a constant-slope ocean, J. Fluid Mech., 535, 33-64, 2005.

Titov, V. V. and Gonzalez, F. I.: Implementation and testing of the method of splitting tsunami (MOST) model, NOAA Technical Memorandum ERL PMEL-112, 1997, available at: http: //www.pmel.noaa.gov/pubs/PDF/tito1927/tito1927.pdf, last access: 2008.

Titov, V. V. and Synolakis, C. I.: Extreme inundation flows during the Hokkaido-Nansei-Oki tsunami, Geophys. Res. Lett., 24, 1315-1318, 1997.

Wood, D. J., Peregrine, D. H., and Bruce, T.: Study of wave impact against a wall with pressure-impulse theory, J. Waterw. Port C.-ASCE, 126, 182-190, 2000.

Yeh, H., Liu, P. L.-F., and Synolakis, C.: Long Wave Runup Models, World Scientific Publishing, Singapore, 1997.

Zahibo, N., Pelinovsky, E., Golinko, V., and Osipenko, N.: Tsunami wave runup on coasts of narrow bays, Int. J. Fluid Mech. Res., 33, 106-118, 2006.

Zelt, J. A.: Tsunamis: the response of harbours with sloping boundaries to long wave excitation, California Institute of Technology, Pasadena, CA, USA, Report No. KH-R-47, 1986. 